\title{
Nutritional evaluation of Jambo forage using near infrared reflectance spectroscopy and comparison with wet chemistry analysis
}

\author{
ZH Khandaker and ABM Khaleduzzaman
}

Department of Animal Nutrition, Bangladesh Agricultural University, Mymensingh 2202

\begin{abstract}
A study was undertaken for the nutritional evaluation of Jambo forage by using Near Infrared Reflectance Spectroscopy (NIRS) and compare with the values obtained from wet chemistry analysis. Near infra-red reflectance spectrum of ground forage samples were obtained in duplicate (scanning number 32, resolution 8) with an FT-NIRS (Bruker, MPA, Germany) systems monochromator (700-2400 $\mathrm{nm}$ ) using a Qurtz cup sampling device. For the development of local calibration equations, multivariate analysis was performed by a commercial analysis program Optical User Software (OPUS) and OpusLab to relate the spectral data and corresponding concentration values for each nutrient component of forage. The 108 Jambo forage samples were collected from 108 cultivated experimental plots and ground through $2.0 \mathrm{~mm}$ screen for analysis the proximate components (Moisture, CP, CF, NFE and ash). The value for each component was placed into calibration group for NIRS equation development. The root mean square error of estimation (RMSEE) for the determination of CP, CF, NFE and total ash of Jambo forage was $0.33,0.51,1.14$ and $0.39 \%$ respectively with correlation coefficient $(r 2)$ of $79.18,82.04$, 87.92 and 84.37 respectively. After cross validation, the root mean square error cross validation (RMSECV) for the CP, CF, NFE and total ash of Jambo forage were $0.37,0.58,1.41$ and $0.48 \%$ respectively with correlation coefficient $(r 2)$ of $72.42,73.85,78.87$ and 73.78 respectively. The mean predicted values of $\mathrm{CP}, \mathrm{CF}, \mathrm{NFE}$ and total ash by NIRS are close to the mean laboratory values determined by wet chemistry analysis. It can be concluded that NIRS could potentially be used to predict the nutritional quality of Jambo forage.
\end{abstract}

Key words: Evaluation, near infrared reflectance spectroscopy, wet chemistry,

Bangladesh Animal Husandry Association. All rights reserved.

Bang. J. Anim. Sci. 2011. 40 (1-2): 46-50

\section{Introduction}

Green forages are usually the cheapest source of feed nutrients required by dairy animals for growth, body maintenance and milk production. Forage quality is best defined in terms of the value of the forage in production of milk, meat and wool, when fed to animals, but the time and cost of large animal feeding trials limit their use in routine analysis of forage samples. Wet chemistry (chemical composition) can provide accurate analyses of laboratory estimates of nutritive value; however, an extended period can elapse between the time a sample is submitted for analysis and the time when results are returned and are also expensive method. It would be beneficial in feeding program to have a rapid method that would allow evaluating the forage samples.
Near Infrared Reflectance Spectroscopy (NIRS) is a rapid screening tool to evaluate the chemical composition and nutritive value of forages and requires no chemical reagents. Once calibrations are in place, it takes just a minute to have the results of one or more constituents which by conventional chemistry may take hours or days. Because of its rapid analysis time, and the capability of performing several analyses simultaneously, interest in utilizing NIRS in determining forage quality has increased. In many countries NIRS has been employed in university extension forage testing program (Martin and Linn 1985 and Jones et al. 1987) and by commercial laboratories (Meyer 1985). Norris et al. (1976) successfully developed NIRS equations to predict forage quality and the correlation coefficients were 0.99 for $\mathrm{CP}, 0.98$ for NDF, 0.96 for ADF, 0.96 for lignin, 0.95 for IVDMD, 0.88 for DMD, 0.80 for DMI and 0.85 for 
digestible energy intake (DEI). The standard error of analysis (SEA) was $\pm 0.95 \%$ for $C P$, $\pm 3.1 \%$ for NDF, $\pm 5.1 \%$ for DMD and $\pm 7.9 \mathrm{~g}$ for DMI. Utilizing a combination of wet chemistry and NIRS analysis, Brown and Moore (1987) found standard error of calibration (SEC) and analysis (SEA) ranging from 0.14 to $0.79 \%$ and 0.32 to $0.83 \%$ respectively for NIRS analysis of CP Although many countries are successfully applied NIRS to predict nutritional quality of feeds and forages but the concept of NIRS for the determination of forage quality is still new in Bangladesh. Since the concept of NIRS is still new in Bangladesh but a few commercial laboratories is going to utilize NIRS in determining forage quality. However, little is known about the potential of NIR spectroscopy for the nutritional evaluation of locally available forages in Bangladesh. Therefore, the present investigation was undertaken to estimate the chemical composition of forage samples by NIRS and compare with those of the forage as determined by wet chemistry analysis in order to find the feasibility of using NRIS in predicting the chemical composition forages in the country.

\section{Materials and Methods}

\section{Sample collection and preparation}

A total of 108 Jambo forage samples (used in wet chemistry analysis) were collected and samples were ground to pass through $2.0 \mathrm{~mm}$ screen for the homogeneous particle size using Cemotec Grinding Mill (Foss Tecator, Sweden).

\section{Spectroscopic and laboratory analysis of sample}

Identification of appropriate samples was the first step in utilizing a combination of NIRS and wet chemistry analysis to develop calibration equations and the validation of calibration equations for predicting the nutritional quality of forage samples by near infrared reflectance spectroscopy. The ground forage samples (used in wet chemistry) were scanned in duplicate (scanning number 32, resolution 8) with an FTNIRS (Bruker, MPA, Germany) systems monochromator (700-2400 nm) using a Qurtz cup sampling device. The entire spectrum was stored automatically in respective folder. The proximate components (Moisture, $\mathrm{CP}, \mathrm{CF}$, NFE and total ash) were determined by the procedure of AOAC (2000).

\section{Development of calibration model}

For the development of calibration model in the present experiment, multivariate analysis was performed by a commercial analysis program Optical User Software (OPUS) and OpusLab provided by Bruker, MPA, Germany to relate the spectral data and corresponding concentration values for each nutrient component ( $\mathrm{CP}, \mathrm{CF}$, NFE and total ash) of forage samples. The model was developed using Partial Least Squares (PLS) algorithm and the spectral data were processed by a suitable mathematical method e.g. first derivative, second derivative, vector normalization, subtraction of straight line. Appropriate frequency range of the spectrum was selected to get good correlation between the changes in spectral and the concentration data.

\section{Validation of the model}

The suitability of the chosen data processing method and the frequency range for method development was evaluated during validation. In the case of cross validation, individual samples were taken from the calibration set. Using the remaining samples, a calibration model was established and used to analyze the previously extracted samples. This procedure of removing samples, analyzing them, and returning them to the calibration data set was continued successively until all the samples had been analyzed once A comparison of the resulting analysis values with the original raw data allowed the calculation of the predictive error of the complete data system, the root mean square error cross validation (RMSECV). The Root Mean Square Error of Estmation values of nutrient component determined by analytical laboratory versus predicted values by the NIRS calibration was calculated to give the accuracy of the model. During the validation, potential outliers could be detected easily and only after all outliers had been removed from calibration data set, and finally after the optimum parameters had been found, the calibration model was established.

\section{Results and Discussion}

\section{Chemical composition of forage by NIRS}

The Near Infrared Reflectance Spectroscopy statistics values for the prediction of $\mathrm{CP}, \mathrm{CF}, \mathrm{NFE}$ and total Ash (on DM basis) of Jambo forage samples are shown in Table 1. 


\section{Evaluation of Jambo forage}

\section{Prediction of CP in forages}

The mean predicted value of CP of Jamboo forage was 6.82 , which was very much close to the wet chemistry value of 6.81 . In addition, the resulted correlation coefficient ( $r 2$ ) was 79.18 in developing calibration model for forage by NIRS indicating that the mentioned mathematical models were closely related to the wet chemistry (Kjeldahl procedure) values with a high degree of linearity. The standard error of calibration (RMSEE) was $0.33 \%$. The number of PLS factors in predicting CP of Zambo forages were 4 to get the lowest standard error of cross validation (RMSECV) which was $0.37 \%$ and correlation coefficient ( $r 2$ ) was 72.42 (Figure 1a). The results are in agreement with the findings of Brown and Moore (1987) who reported that the standard error of calibration (SEC) ranged from 0.14 to $0.79 \%$ while those for standard error of analysis or prediction (SEA) ranged from 0.32 to $0.83 \%$ after validation in the analysis of $\mathrm{CP}$ of forage samples through NIRS. These values were similar to or lower than those reported for CP by Barton and Burdick (1981) and Shenk et al. (1981). Nelson and Montgomery (1983) observed SEC and SEA values ranging from 1.13 to $1.21 \%$ and 1.08 to $1.20 \%$ respectively for NIR analysis of CP value in tropical forage. The PLS factors in the analysis of CP in Jambo samples was only 4 . In PLS regression, the spectral data and the concentration data are first encoded in a matrix form and then reduced to only a few factors (rank). If too many factors are chosen, the model tries to account even the smaller changes in data set which creates spectral noise ("overfitting"). In this case, spectral information, unspecific for the sample is included in the model and the deterioration of the analysis results is also to be expected from the model (Conzen 2003). Therefore, the factors with lowest RMSECV determined by cross validation 4 would be optimum for the prediction of CP in forage.

\section{Prediction of CF in forages}

The mean predicted CF (\%) value of forage using NIRS was 30.47, which was very much closer to the wet chemistry value of 30.46 . The standard error (SE) of calibration was $0.51 \%$ with correlation coefficient $\left(r^{2}\right)$ of 82.04 for the prediction of CF in Jambo forage samples. The SE after cross validation for the prediction of CF in Zambo forage samples was $0.58 \%$ which was similar to those reported by Brown et al. (1987). The model developed for the prediction of CF in Zambo forages in the present experiment appears to be sufficiently accurate for quality control applications. In addition, the correlation coefficient $\left(r^{2}\right)$ after cross validation of Zambo forage was 73.45 indicating the accuracy of the model.

\section{Prediction of NFE in forages}

The mean value of NFE in Jambo forage predicted by using NIRS was 30.47 , which was very much closer to the NFE value (30.46) that as was determined in the laboratory. The standard error (SE) of calibration was $1.14 \%$ with correlation coefficient $\left(r^{2}\right)$ of 87.92 for the prediction of NFE in Zambo forage samples. The RMSECV for the prediction of NFE in Jambo forage samples $(1.41 \%)$ was similar to those reported by Brown and Moore (1987). The model developed for the prediction of NSC (Non-structural carbohydrates) supports to the findings of the present experiment which appears to be sufficiently accurate for quality control applications. In addition, the correlation coefficients $\left(r^{2}\right)$ after cross validation of Zambo forage was 78.87 also indicating the accuracy of the model.

\section{Prediction of total ash in forage}

Mineral analysis of forages by NIRS appears unlikely as NIRS do not absorb energy in the NIRS region. However, correlations between minerals and other organic components allow reasonable NIR calibrations to be obtained in some cases. In the present study, the mean value of total ash (\%) contents in Zambo forage predicted by using NIRS was 7.04, which was similar to the total ash (7.08) that was determined in the laboratory. The standard error (SE) of calibration was $0.39 \%$ with correlation coefficient $\left(r^{2}\right)$ of 84.37 for the prediction of total ash in forage. The SE after cross validation for the prediction of total ash in forage sample was $0.48 \%$ with correlation coefficient 73.78 indicated that the NIRS could potentially be used in minerals analysis. Smith and Flinn (1991) obtained a calibration for $\mathrm{Mg}$ in perennial ryegrass with a coefficient of variation (CV) of 16 and concluded that NIRS could be a useful tool for preliminary screening of ryegrass lines for $\mathrm{Mg}$ in plant breeding program. 
Table 1. Laboratory analytical values, NIRS values of $C P, C F, N F E$ and total ash (on DM basis) and the relevant NIRS statistics of Jambo forage samples

\begin{tabular}{|l|c|c|c|c|c|c|c|c|c|}
\hline $\begin{array}{c}\text { Items } \\
(\%)\end{array}$ & $\begin{array}{c}\text { No. of } \\
\text { samples }\end{array}$ & $\begin{array}{c}\text { Laboratory } \\
\text { values }\end{array}$ & $\begin{array}{c}\text { NIRS values } \\
\text { (predicted) }\end{array}$ & \multicolumn{2}{c|}{ Cross validation statistics } & \multicolumn{3}{c|}{ Calibration statistics } \\
\hline & & Mean & Mean & PLS $^{1}$ & RMSECV $^{2}$ & $r^{2}$ & PLS & RMSEE $^{3}$ & $r^{2}$ \\
\hline CP & 108 & 6.81 & 6.82 & 4 & 0.37 & 72.42 & 4 & 0.33 & 79.18 \\
\hline CF & 108 & 30.46 & 30.47 & 8 & 0.58 & 73.45 & 8 & 0.51 & 82.04 \\
\hline NFE & 108 & 53.31 & 53.33 & 10 & 1.41 & 78.87 & 10 & 1.14 & 87.92 \\
\hline Total ash & 108 & 7.08 & 7.04 & 10 & 0.48 & 73.78 & 10 & 0.39 & 84.37 \\
\hline
\end{tabular}

${ }^{1} P L S$, partial least square, ${ }^{2} R M S E C V$, root mean square error cross validation; ${ }^{3} R M S E E$, root mean square error of estimation or standard error of calibration (SEC); $r^{2}$, correlation coefficient.

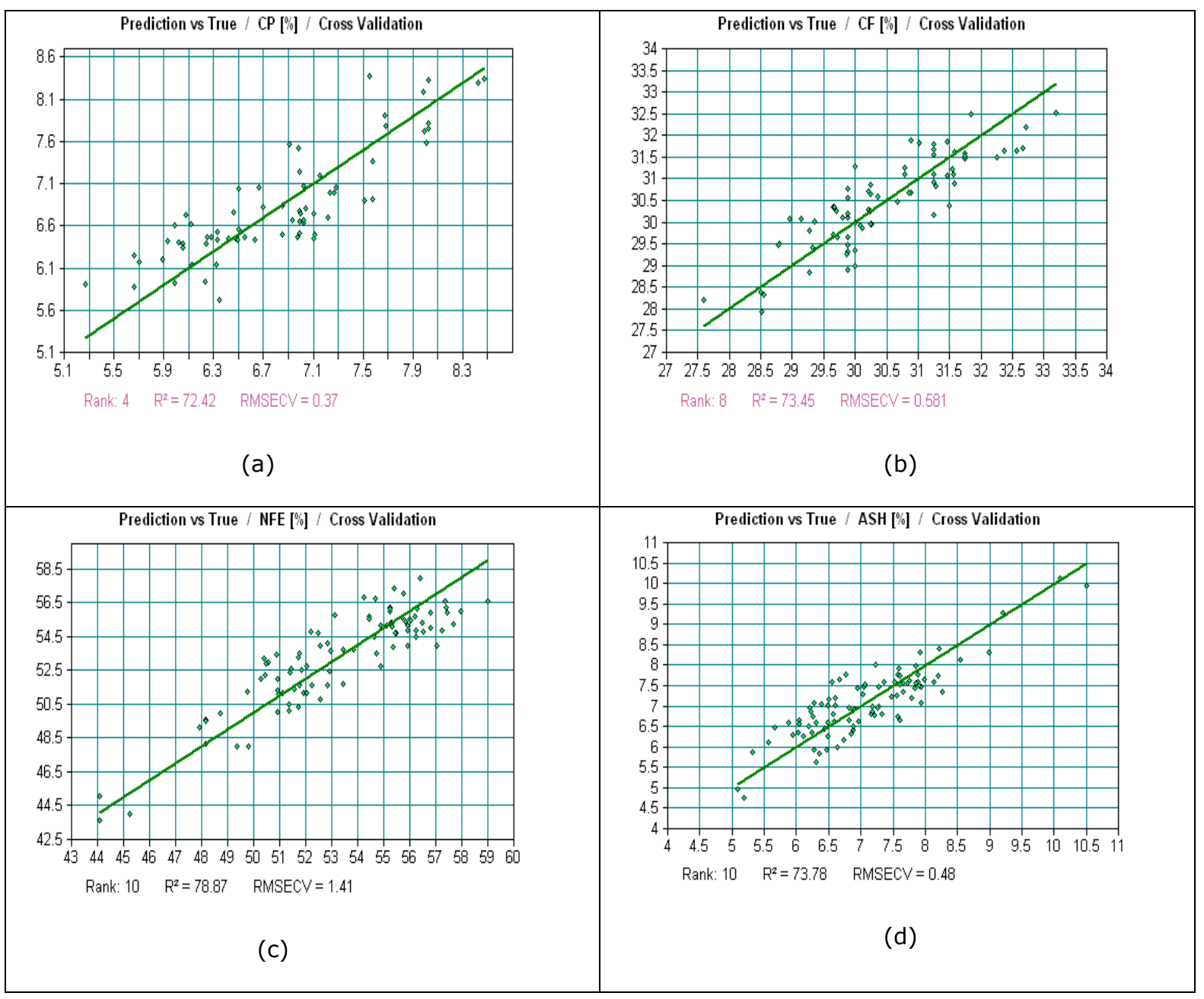

Figure 1. Regression equations of prediction vs true (laboratory) values for $C P(a), C F(b), N F E(c)$ and total ash(d) determination in Zambo forage samples by NIRS

\section{Conclusion}

The mean predicted values of $\mathrm{CP}, \mathrm{CF}, \mathrm{NFE}$ and total ash of Jambo forage samples by NIRS are very close to those determined by the wet chemistry analysis. In addition the values of $\mathrm{CP}$, $\mathrm{CF}, \mathrm{NFE}$ and total ash between methods were significantly correlated. Therefore, NIRS could efficiently be used to predict the nutritional quality of Jambo forage. Further research should be conducted by using a large number of forage samples for final recommendation.

\section{Acknowledgements}

The author is grateful to the University Grants Commission of Bangladesh, for providing fund for this study. The help and cooperation of the Head of the Department of Animal Nutrition, BAU, Mymensingh is also acknowledged for providing 


\section{Evaluation of Jambo forage}

the necessary facilities for this work. Finally, the author expresses his thanks to Advanced Laboratories, Advance Animal Science Co. Ltd. for giving permission to use their valuable NIRS machine and cordial assistance of laboratory staffs.

\section{References}

AOAC (2000). Official Methods of Analysis. $17^{\text {th }}$ Ed. Association of Official Agricultural Chemists Washington, DC. P. 1045-1052.

Barton FE and D Burdick (1981). Prediction of forage quality with NIR reflectance spectroscopy. In: Proc. $14^{\text {th }}$ International Grassland Congress.p. 532. Westview Press Boulder Co. USA.

Brown WF and JE Moore (1987). Analysis of forage research samples utilizing a combination of wet chemistry and near infrared reflectance spectroscopy. J. Anim. Sci. 64: 271-282.

Conzen JP (2003). Multivariate Calibration. A practical guide for developing methods in the quantitative analytical chemistry. $1^{\text {st }}$ English edition, translated from the $3^{\text {rd }}$ German edition. Bruker Optick, GmbH.

Jones GM, NS Wade, JP Baker and EM Ranck (1987). Use of near infrared reflectance spectroscopy in forage testing. J. Dairy Sci. 70: 1086

Matin NP and JG Linn (1985). Extension applications in NIRS technology transfer.
In: G.C. Marten, J.S. Shenk and F.E. Barton, II (Ed.) Near infrared reflectance spectroscopy (NIRS): Analysis of forage quality. P. 48-53. ARS, USDA, Washington, DC.

Meyer DW (1985). Use of NIRS in commercial testing laboratory. Agron. Abstr. p 127. American Society of Agronomy. Madison, WI.

Nelson BD and CR Montgomery (1983). Prediction of bermudagrass and bahiagrass forages composition and digestibility with a near-infrared multiple filter spectrophotometer. Annual Progress Report. Southeast Research Station. Louisiana State University. P. 183.

Norris KH, RF Barnes, JE Moore and JS Shenk (1976). Predicting forage quality by near infrared reflectance spectroscopy. Journal of Animal Science 43: 889

Shenk JS, I Landa, MR Hoover and MO Westerhaus (1981). Description and evaluation of near infrared reflectance spectro-computer for forage and grain analysis. Crop Sci. 21: 355-358.

Smith KF and PC Flinn (1991). Monitoring the performance of a broad-based calibration for measuring the nutritive value of two independent populations of pasture using near infrared reflectance (NIR) spectroscopy. Australian J. Exp. Agric. 31: 205210. 